\title{
Epidermolysis Bullosa Dystrophica, Hypoplastic Type, Associated with Pelger-Huet Anomaly
}

\author{
I. CAT, LEIDE P. MARINONI, D. J. GIRALDI, V. de PAULA FURTADO, \\ R. PASQUINI, N. FREIRE-MAIA, and H. BRAGA
}

From the Departamento de Pediatria and Departamento de Clinica Médica (Hematologia) do Hospital de Clinicas and from the Laboratório de Genética Humana, Universidade Federal do Paraná, Curitiba, Paraná, Brazil

A 7-year-old boy with epidermolysis bullosa dystrophica, hypoplastic type, and the PelgerHuet anomaly is described.

\section{Case Report}

At birth a diagnosis of epidermolysis bullosa dystrophica was suggested by absence of skin in the left pretibial region, and shortly afterwards bullae appeared in the axillary, inguinal, and cervical regions, on the face and on the buccal and pharyngeal mucous membranes. At the age of 12 months atrophic lesions appeared in the nails with resultant loss. The child also had difficulty in swallowing and bouts of constipation.

On examination at 7 years this underdeveloped child had many features characteristic of epidermolysis bullosa. He had abnormal facial hair, his mouth was small, and mucous membranes were atrophic and hyperaemic. The tongue was also atrophic and ulcerated and protrusion was impossible. The teeth also showed atrophic changes. Desquamating atrophic erythematous lesions were plentiful on many parts of the body. The extremities were cold, the fingers were partially flexed due to scarring following bullae. The skin over the feet was paper-like and the terminal portion of the first left toe was missing (Fig. 1). All finger- and toe-nails had been lost. Radiology showed partial bone resorption of the distal phalanges of the fingers and toes. Peripheral blood examination revealed the presence of the PelgerHuet anomaly (Fig. 2).

This child is the younger of two sibs. The parents were not related. There was no family history of epidermolysis bullosa dystrophica, but on investigation, leucocytes of the father, a paternal aunt, and the paternal grandmother of the propositus all showed the typical Pelger-Huet changes.

\section{Comment}

Epidermolysis bullosa dystrophica is inherited as an autosomal recessive trait, while the Pelger-Huet anomaly is inherited as an autosomal dominant

Received March 9, 1967. condition. The case reported appears to be the first in which both these conditions have been found in the same individual.

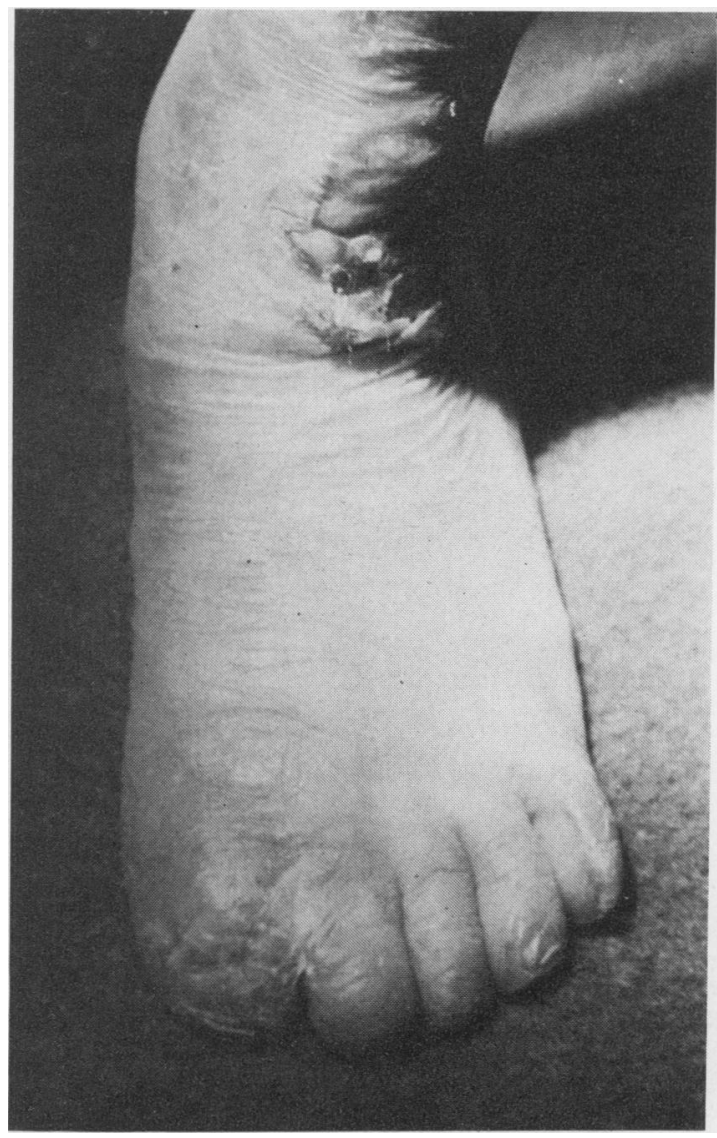

Fig. 1. The foot, showing paper-like skin, loss of terminal portion of big toe, and no toe-nails. 


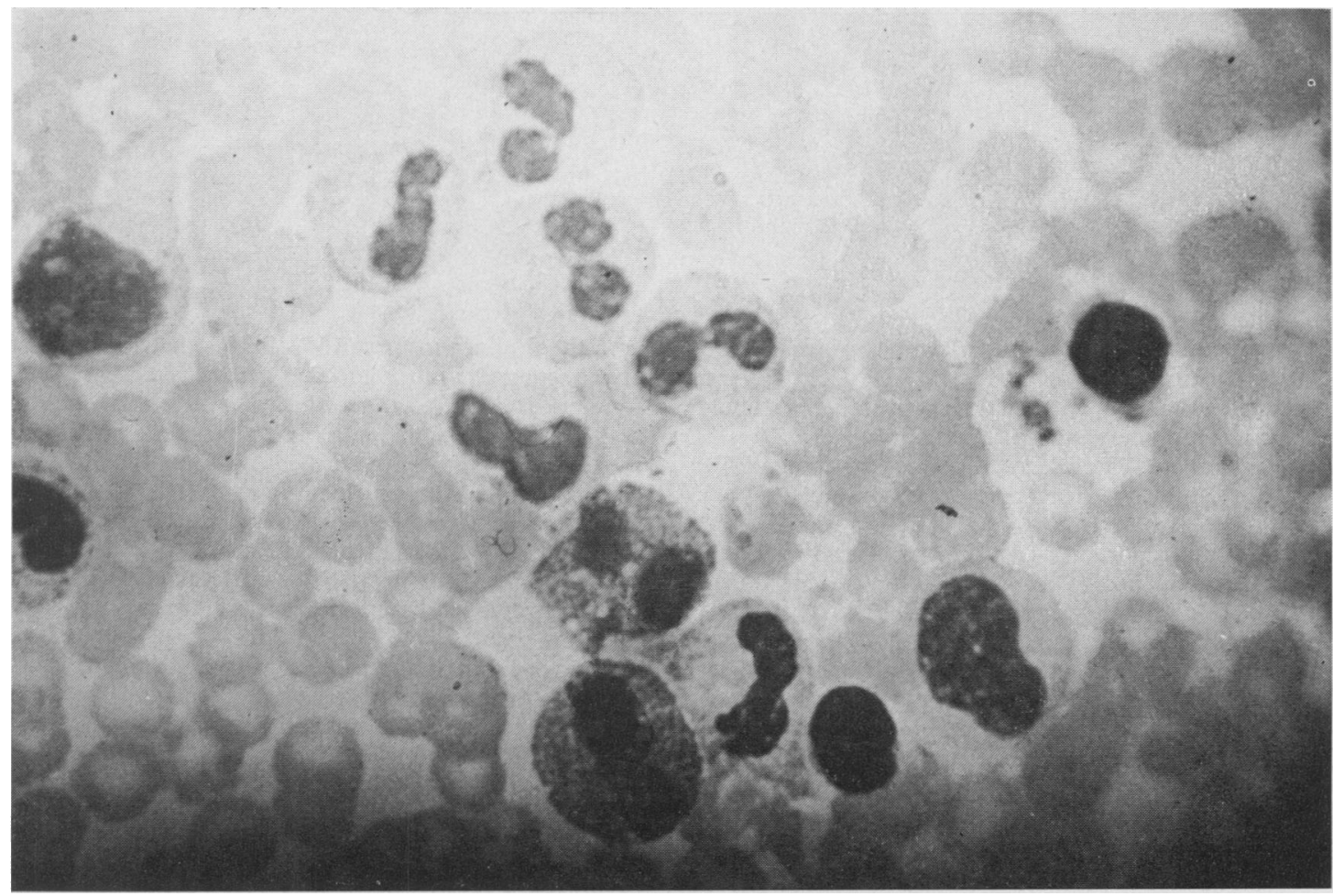

FIG. 2. Peripheral blood examination showing Pelger-Huet anomaly. $(\times 1500$.

\section{Summary}

A boy is described with the hypoplastic type of epidermolysis bullosa dystrophica and the PelgerHuet anomaly. Both entities presented the classical clinical and haematological findings.
This research was supported by grants from the Reseach of the Federal University of Paraná, the Rockefeller Foundation, and the Brazilian National Research Council. 